\title{
COMPLEMENTARIEDAD ENTRE GENERACIÓN HIDROELÉCTRICA Y FOTOVOLTAICA. UN BREVE ANÁLISIS ECONÓMICO
}

\section{RESUMEN}

La generación fotovoltaica, si bien ha tenido un desarrollo favorable en cuento a costos, presenta el inconveniente de que solo genera electricidad durante el día. Este artículo muestra cómo podrían complementarse la energía fotovoltaica con las hidroeléctricas que poseen represamiento de agua.

JEL: L94; N76; Q52

PALABRAS CLAVES: Energía fotovoltaica; economía medioambiental; control de costos

\begin{abstract}
Solar power generation has shown significant advances by reducing generation costs. However it has the inconvenience that it only generates electricity during day hours. This paper shows that the problem can be solved by complementing solar generation with hydroelectric power.
\end{abstract}

JEL: L94; N76; Q52

KEYWORDS: Photovoltaic Energy, Environmental Economics, Cost Control

\footnotetext{
A Investigador docente de la Escuela Profesional de Economía de la Facultad de Ciencias Contables, Económicas y Financieras de la Universidad de San Martín de Porres. Se agradece enormemente la asistencia de investigación de Krisell Cainicela y Ricardo Rojas quienes han realizado un excelente trabajo.
} 


\section{LAS ENERGÍAS RENOVABLES NO CONVENCIONALES (RER) Y EL EFECTO INVERNADERO}

La producción de bienes y servicios requiere de energía. Por lo tanto, conforme se incrementa el consumo mundial de estos aumentan los requerimientos de energía. La Agencia Internacional de Energía considera que,

[...] mantener la producción de energía a este ritmo de crecimiento por medios convencionales es insostenible tanto por el incremento de precios de energía como por las emisiones de efecto invernadero incluyendo $\mathrm{CO} 2$, (dióxido de carbono) las cuales incrementarían la temperatura terrestre en 6 grados centígrados en el largo plazo." $1 /$.

Por ello, teniendo como objetivo que el incremento de temperatura a nivel mundial sea de sólo 2 grados centígrados, dicha agencia recomienda:

- Empleo de incentivos para reducir la emisión de gases de efecto invernadero poniendo precios a la emisión de los mismos (carbono entre otros).

- Esfuerzos para promover investigación y desarrollo con objeto de mejorar la performance y reducir el costo de nuevas tecnologías no o menos contaminantes.

- Introducción de incentivos económicos para promover tecnologías menos contaminantes.

- Aplicación de estándares y códigos de performance en las tecnologías de producción de energía.

- Otorgamiento de beneficios impositivos por parte de los gobiernos para reducir los costos de capital de nuevas tecnologías no contaminantes.

- Implementación de políticas de gobierno con una visión de sistema incluyendo incentivos y reglas para el desarrollo y operación de infraestructura (tales como redes inteligentes que permitan operar de manera flexible el manejo de las energías no convencionales).

Estas medidas se ven reflejadas en los acuerdos de Kioto (1997), Copenhague (2009), Durban (2011), y Paris (2015). Por otra parte, siendo el clima un bien público justifica la intervención del estado con el fin de equilibrar los beneficios y costos privados con los públicos.

\section{a. Situación actual y problemas}

La política energética del Perú, dentro de la política internacional de promoción de energías limpias es promover energías renovables no convencionales. El mecanismo utilizado está basado en el Decreto Ley 1002 de 2018. Según este decreto el Estado a través del Ministerio de Energía y Minas (MINEM) adjudica cada año a través de subastas un porcentaje de las necesidades de electricidad hasta alcanzar el $5 \%$ de capacidad del sistema eléctrico con estas tecnologías. La capacidad adjudicada a la fecha se muestra en el cuadro 1.

\section{LAS ENERGÍAS RENOVABLES NO CONVENCIONALES (RER) Y EL EFECTO INVERNADERO}

La producción de bienes y servicios requiere de energía. Por lo tanto, conforme se incrementa el consumo mundial de estos aumentan los requerimientos de energía. La Agencia Internacional de Energía considera que,

[...] mantener la producción de energía a este ritmo de crecimiento por medios convencionales es insostenible tanto por el incremento de precios de energía como por las emisiones de efecto invernadero incluyendo $\mathrm{CO} 2$, (dióxido de carbono) las cuales incrementarían la temperatura terrestre en 6 grados centígrados en el largo plazo." $1 /$.

Por ello, teniendo como objetivo que el incremento de temperatura a nivel mundial sea de sólo 2 grados centígrados, dicha agencia recomienda:

- Empleo de incentivos para reducir la emisión de gases de efecto invernadero poniendo precios a la emisión de los mismos (carbono entre otros).

- Esfuerzos para promover investigación y desarrollo con objeto de mejorar la performance y reducir el costo de nuevas tecnologías no o menos contaminantes.

- Introducción de incentivos económicos para promover tecnologías menos contaminantes.

- Aplicación de estándares y códigos de performance en las tecnologías de producción de energía.

- Otorgamiento de beneficios impositivos por parte de los gobiernos para reducir los costos de capital de nuevas tecnologías no contaminantes. 
- Implementación de políticas de gobierno con una visión de sistema incluyendo incentivos y reglas para el desarrollo y operación de infraestructura (tales como redes inteligentes que permitan operar de manera flexible el manejo de las energías no convencionales).

Estas medidas se ven reflejadas en los acuerdos de Kioto (1997), Copenhague (2009), Durban (2011), y Paris (2015). Por otra parte, siendo el clima un bien público justifica la intervención del estado con el fin de equilibrar los beneficios y costos privados con los públicos.

\section{a. Situación actual y problemas}

La política energética del Perú, dentro de la política internacional de promoción de energías limpias es promover energías renovables no convencionales. El mecanismo utilizado está basado en el Decreto Ley 1002 de 2018. Según este decreto el Estado a través del Ministerio de Energía y Minas (MINEM) adjudica cada año a través de subastas un porcentaje de las necesidades de electricidad hasta alcanzar el $5 \%$ de capacidad del sistema eléctrico con estas tecnologías. La capacidad adjudicada a la fecha se muestra en el cuadro 1.

\section{Cuadro 1. Capacidad adjudicada en subastas de energias renovables no tradicionales (RER) hasta 2019.}

\begin{tabular}{|c|c|}
\hline Tecnología & Capacidad Megawatts (MW) \\
\hline Biomasa & 23 \\
\hline Biogas & 11 \\
\hline Eólica & 394 \\
\hline Solar & 280 \\
\hline Minihidro & 566 \\
\hline TOTAL & 1274 \\
\hline
\end{tabular}

Desde el aspecto de costos de electricidad con tecnologías RER, éstas se han vuelto competitivas con el transcurrir de las subastas: el precio de la energía eólica se redujo de US\$80.4/MWh en 2009 a US\$37.7/MWh en 2013, y el precio de la energía solar se redujo de US\$221.1/MWh en 2009 a US\$37.7/MWh en 2013, valores inferiores a los US\$45/MWh a US\$60/MWh de las energías de generación a gas natural e hidroeléctricas. Sin embargo, el problema de las energías eólica y solar es la intermitencia. Debido a ello, cuando estas energías dejan de producir se debe recurrir a otro tipo de generación eléctrica lo cual genera un costo adicional por tener que mantener estas últimas como respaldo.

a. Opciones para evitar la afectación al sistema eléctrico por la intermitencia de las RER

El problema de intermitencia de las RER se puede resolver mediante: (a) un sistema que permita tener generadores convencionales de reserva; y (b) almacenamiento de electricidad. El primer caso resultaría en altos costos para el sistema eléctrico por tener unidades sin operar y costos de operación elevados en el momento en que estas unidades entraran para suplir la energía no provista por las RER. El segundo caso podría consistir en baterías para almacenar electricidad o reservorios de agua de centrales hidroeléctricas que serían empleados por ésta cuando no operaran las RER. Actualmente, las baterías son costosas, del orden de US\$96/MWh, (Megawatts - hora) aunque se espera que bajen a US\$70 por MWh según Bloomberg New Energy Finance (BNEF) (www.pv-magazine-latam. com). En cambio, la alternativa de complementar RER con hidroeléctricas las cuales acumularían agua en sus represas cuando operan las RER para luego utilizar dicha agua para generación hidroeléctrica en horas en que la generación RER está indisponible podría ser la mejor opción. Si bien en el Perú las hidroeléctricas ya están actuando de esta manera, el costo que esto representa para el sistema no ha sido estudiado. Este análisis es el objeto del presente artículo.

\section{OBJETIVO DE LA INVESTIGACIÓN}

En línea con la práctica actual observada se propone elaborar un modelo que estudie la complementariedad entre energía hidroeléctrica $\mathrm{y}$ fotovoltaica y desarrollar reglas para optimizar esta complementariedad.

La complementariedad entre hidroelectricidad y energía eléctrica solar fotovoltaica es como sigue: durante las horas de producción de energía solar, las hidroeléctricas usan menos agua represada la cual utilizan cuando las fotovoltaicas no producen energía eléctrica. El gráfico 1 de complementariedad es como sigue (ver Dammert et. al., El futuro de las energías renovables en el Perú, Fondo Editorial de la Universidad de San Martín de Porres, en publicación) 


\section{Gráfico 1. Complementariedad entre hidroelectricidad y energia solar fotovoltaica}

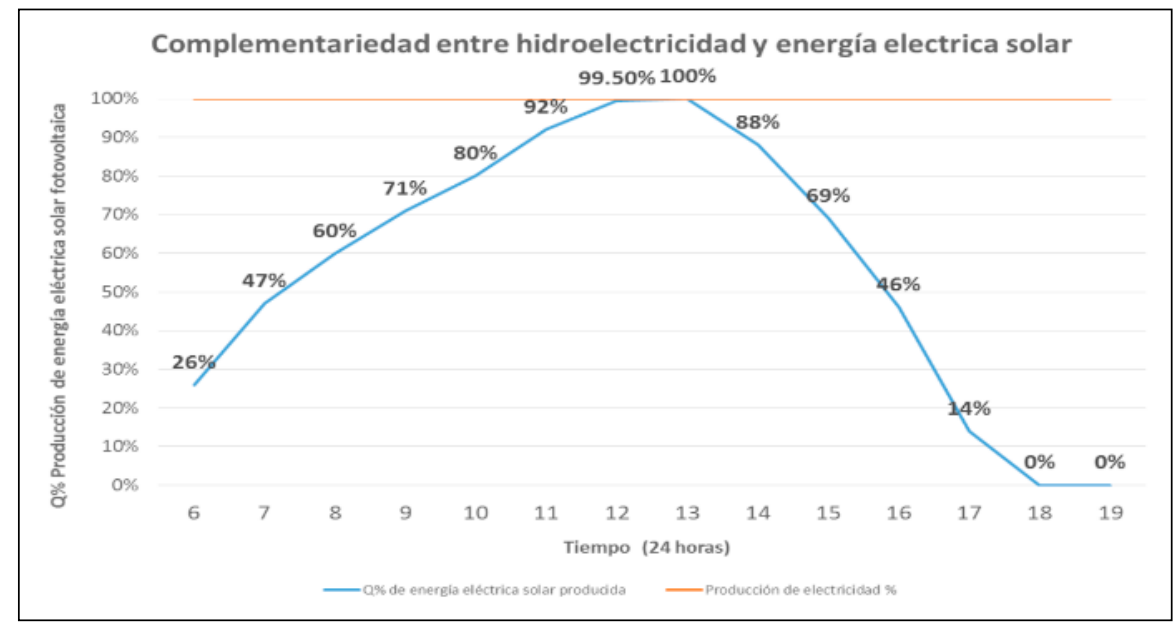

Elaboración: Cálculos del autor

El modelo considera la producción de energía solar de 6 am a $7 \mathrm{pm}$ y la cantidad de hidroelectricidad para complementar considerando la diferencia entre la máxima producción de la solar y la producción en el tiempo t según la fórmula:

Ehidro(t) $=$ Max prod solar - Prod. Solar (t) (1)

Donde:

- Ehidro $(\mathrm{t})$ : producción de hidroelectricidad en tiempo ( $\mathrm{t}$ ) en MWh.
- Max prod solar: punto máximo de prod de energía solar para una generadora

- Fotovoltaica típica en MWh.

- Prod. Solar: producción de la generadora Fotovoltaica para el tiempo t en

- MWh

Un histograma que muestra dicha complementariedad se muestra en el Gráfico 2.

\section{Gráfico 2. Complementariedad entre hidroelectricidad y energia solar fotovoltaica}

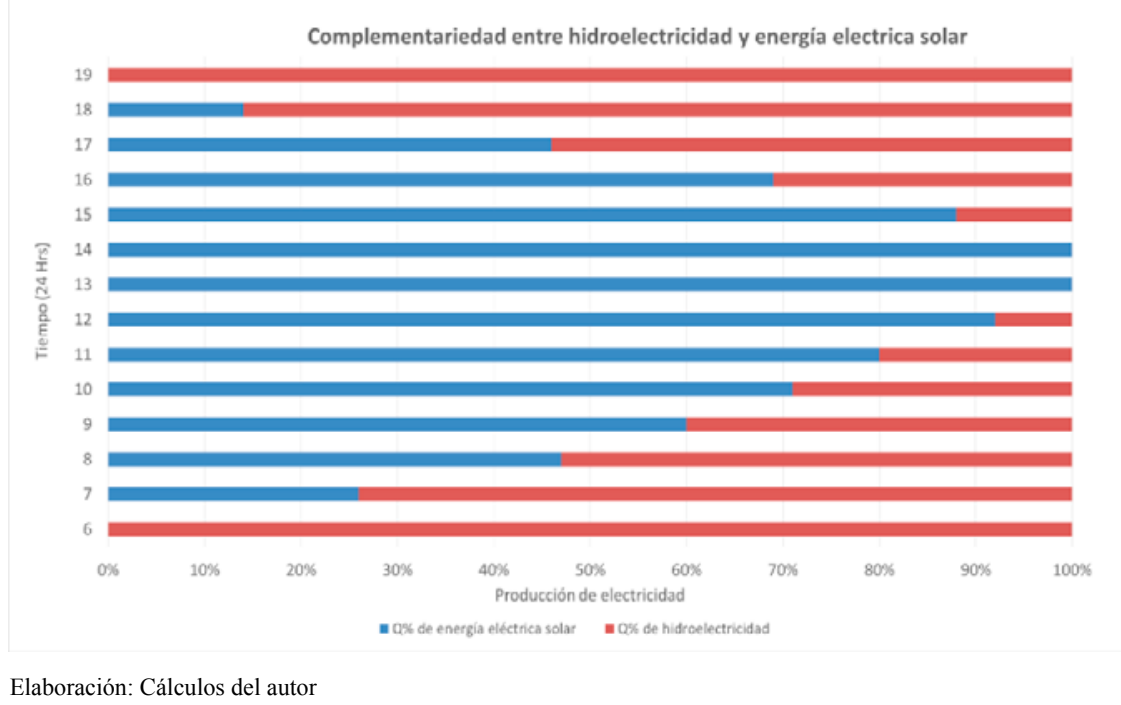

Del Gráfico 1, se estimará una función de producción de energía solar para las horas t=1,..24. Esta se desarrollará tanto en forma gráfica como por medio de una ecuación. 
La función obtenida y sus desviaciones versus los valores observados son como sigue:

$\mathrm{Y}=12.7142857143+23.0164835165 * \mathrm{X}-1.78571428571 * \mathrm{X}^{\wedge} 2$ Gráfico 3. Función de complementariedad entre hidroelectricidad y energia solar
fotovoltaica

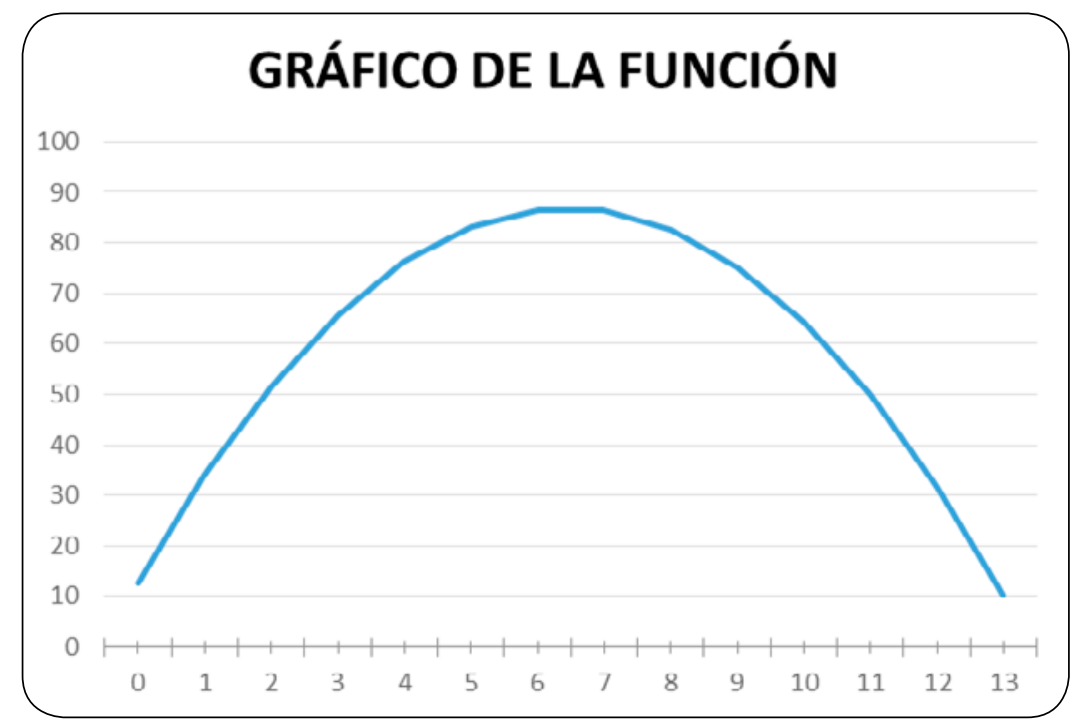

Elaboración: Cálculos del autor

Con esta ecuación se puede calcular cuánto se requiere producir de hidroelectricidad para abastecer un total de 100kMWh por cada hora con objeto de complementar la producción fotovoltaica. Los resultados son:

$\int_{1}^{14}\left(12.7+23 X-1.786 x^{2}\right) d x=775$

Área de 0 a $24 \times 100=2400$ (producción total)

De estos resultados se tiene que la producción hidroeléctrica es $2400-775=1625$.

O sea, el porcentaje de producción de cada tipo de energía es $32.3 \%$ fotovoltaica y $67.7 \%$ hidroeléctrica.

Si la energía requerida es de 100 y en un día se produce energía solar por 32.3 y energía hidroeléctrica por 67.7 se deben calcular los costos de tener una generadora eléctrica para producir 100, una represa de agua para abastecer 67.7, y una generadora solar para producir 32.3.

1. Por otro lado si sólo se producen los 100 con hidroeléctricas se deben tener los costos de tener una generadora hidroeléctrica para producir 100 y una represa para producir 100.
2. Comparando 1 y 2 se puede establecer la diferencia de costos para producir 100 con y sin fotovoltaicas.

3. Una primera aplicación será obtener costos típicos para 10 y 11. El costo típico de generar con hidroeléctricas es de USc5.5/ kWh de los cuales se estima que el costo de la represa es de entre $40 \%$ y $50 \%$. Por ello, el costo de hidroelectricidad sería entre USc3.3/kWh y USc2.75/kWh y el de la represa entre USc2.2/kWh y USc2.75/ $\mathrm{kWh}$. Por otra parte el costo de generación fotovoltaica es de USc $3 / \mathrm{kWh}$.

a. Si la hidroelectricidad aporta sólo el $67.7 \%$ de lo consumido y la fotovoltaica el

b. $32.3 \%$ de lo consumido, los costos serían:

c. Hidro (la capacidad no cambia) entre USc3.3/kWh y USc $2.75 / \mathrm{kWh}$

d. Represa (67.7\%) entre USc1.49/kWh y USc/kWh1.86

e. Solar USc/kWh USc0.97/kWh

f. TOTAL entre USc5.76/kWh y USc5.58/ $\mathrm{kWh}$

g. Si sólo se produce con hidro el costo es USc $5.5 / \mathrm{kWh}$ 
h. Comparando a con b el producir complementariamente hidros con solares cuesta USc0.26/kWh a USc0.08/ $\mathrm{kWh}$, más que con hidroelectricidad o sea entre $4.5 \%$ y

i. $1.4 \%$ más.

\section{a. Externalidades}

Desde la perspectiva de Helbling (2010) una externalidad negativa se da cuando un contaminador toma decisiones basadas únicamente en el costo y la oportunidad de lucro directos de la producción, sin tener en cuenta los costos indirectos que recaen en las víctimas de la contaminación, como son el deterioro de la calidad de vida y la pérdida de oportunidades de producción. Esto sucede, por ejemplo, cuando una generadora de gas natural produce $\mathrm{CO} 2$ que deviene finalmente en el denominado efecto invernadero.

Enseguida, se presenta una tabla con las principales externalidades veto que se presentan en las diferentes tecnologías, de acuerdo a OSINERGMIN.

\section{Tabla 1: Principales externalidades que se presentan en las diferentes tecnologias}

\begin{tabular}{|c|c|c|}
\hline Tipo de central & $\begin{array}{l}\text { Aspecto ambiental } \\
\text { Impacto ambiental }\end{array}$ & Impacto ambiental \\
\hline \multirow{3}{*}{ Térmica } & $\begin{array}{l}\text { Emisión de gases de combustión (CO2, } \\
\qquad \mathrm{CO}, \mathrm{NOx})\end{array}$ & $\begin{array}{l}\text { Afectación a la salud de las personas. } \\
\text { Perjudica flora y fauna del lugar. } \\
\text { Impacto sobre la calidad de aire, agua y suelo. }\end{array}$ \\
\hline & Emisión de ruido & Afectación a la población, trabajadores y a la fauna del lugar. \\
\hline & Fugas y derrames de hidrocarburos & $\begin{array}{l}\text { Afectación a la salud de las personas. } \\
\text { Perjudica flora y fauna del lugar. } \\
\text { Impacto sobre la calidad de aire, agua y suelo. }\end{array}$ \\
\hline \multirow{3}{*}{ Hidráulica } & $\begin{array}{l}\text { Inundación de grandes áreas para } \\
\text { construcción de embalse }\end{array}$ & $\begin{array}{c}\text { Perdida de suelo fértil } \\
\text { Cambio en la calidad de vida por desplazamiento de la } \\
\text { población. } \\
\text { Desplazamiento de población, cambio en condiciones de vida } \\
\text { e impacto en la salud. } \\
\text { Perjudica flora y fauna del lugar. }\end{array}$ \\
\hline & Emisión de ruido & Afectación a la población, trabajadores y a la fauna del lugar. \\
\hline & $\begin{array}{l}\text { Emisión de gases de efecto invernadero } \\
(\mathrm{CH} 4 \mathrm{Y} \mathrm{CO} 2) \text { por putrefacción } \\
\text { de bosque y subsuelo inundado, y } \\
\text { acumulación de sedimentos en represas }\end{array}$ & $\begin{array}{l}\text { Afectación a la salud de las personas. } \\
\text { Perjudica flora y fauna del lugar. } \\
\text { Impacto sobre la calidad de aire, agua y suelo. }\end{array}$ \\
\hline \multicolumn{2}{|c|}{$\begin{array}{c}\text { Nuclear } \\
\text { Riesgo de explosión nuclear }\end{array}$} & $\begin{array}{l}\text { Peligro de muerte y afectación de la salud de las personas y } \\
\text { trabajadores. }\end{array}$ \\
\hline & Emisión de radiación & $\begin{array}{l}\text { Afectación a la salud de las personas. } \\
\text { Perjudica flora y fauna del lugar. } \\
\text { Inutilización de tierras agrícolas. }\end{array}$ \\
\hline \multirow{2}{*}{ Eólica } & Emisión de ruido & Afectación a la población, trabajadores y a la fauna del lugar. \\
\hline & Instalación de aerogeneradores & $\begin{array}{l}\text { Impacto paisajístico. } \\
\text { Riesgo de colisión de aves. }\end{array}$ \\
\hline
\end{tabular}

Fuente: OSINERMING. 
Las centrales térmicas son las que generan una mayor cantidad de polución, y las centrales hidráulicas, generan más efectos ambientales, debido al tema de traslación de poblaciones y la afectación a la flora y fauna acuática en el área de efecto directo e indirecto del proyecto.

Dentro de las energías renovables no convencionales, las centrales de biomasa, de igual manera generan contaminantes. Las centrales nucleares no emiten contaminantes, pero cabe la posibilidad de impactos ambientales debido al riesgo de fallas en los reactores. Por otro lado, las centrales de biogás y de cogeneración con biomasa generan una externalidad afirmativa al ahorrar emisiones de $\mathrm{CO} 2$.

Al introducirlas externalidades, el costo de generación de las centrales térmicas aumentan en gran medida, mientras que el de la central de biogás permanece constante; las centrales hidráulicas de embalse, las eólicas y las térmicas.

\section{a. Bonos de carbono}

Según el diario Gestión (2013) la empresa Walt Disney compró 437000 bonos de carbono a USD 3.500000 . Se trató de la primera experiencia de un proyecto de esta naturaleza.

Los bonos de carbono se emplean para incentivar a las empresas privadas a utilizar energías limpias a través de subsidios que constituyan la diferencia entre el impacto negativo de emplear un combustible con efecto invernadero versus emplear energías RER.

Según Dammert et al (2017: 345-349) las energías renovables no convencionales son promovidas por la comunidad internacional debido a que su utilización permite reducir $\mathrm{CO} 2$ con lo cual pueden cumplir con sus metas para reducir el impacto del uso de la energía en el calentamiento global. Estas energías no convencionales reemplazarían a las centrales térmicas que tienen efecto invernadero. $\mathrm{Si}$ se considera el costo de las externalidades, el costo de generación de las centrales térmicas aumenta en gran medida, mientras que el costo de las energías renovables permanece igual, con lo cual el costo neto de utilizar estas últimas sería menor.

\section{JUSTIFICACIÓN DEL PAGO ADICIONAL POR LA COMBINACIÓN DE GENERACIÓN SOLAR CON HIDROELÉCTRICA EN VEZ DE ÚNICAMENTE GENERACIÓN HIDROELÉCTRICA}

La European Environment Agency www.eea. europa.eu estimó en 2007 que el costo adicional por contaminación ambiental incluyendo emisiones de efecto invernadero está entre USc $1.3 / \mathrm{kWh}$ y Usc $5 / \mathrm{kWh}$ para generación eléctrica con gas natural y en USc $0.5 / \mathrm{kWh}$ y USc $1 / \mathrm{kWh}$ para generación eléctrica con fotovoltaicas. Por lo tanto la diferencia a favor de fotovoltaicas está entre USc $0.8 / \mathrm{kWh}$ y USc $4 /$ $\mathrm{kWh}$. Estas diferencias son significativamente mayores que los costos adicionales de producir conjuntamente con energía solar e hidroeléctrica mostrados en el párrafo anterior (entre USc0.08/ $\mathrm{kWh}$ y USc0.26/kWh). Ello justificaría un subsidio para la combinación de solares con hidroeléctricas. Alternativamente se podría considerar otorgar bonos de carbono a esta combinación con el objetivo de incentivarla (los bonos de carbono se otorgan a través de diversas instituciones internacionales $\mathrm{y}$ representan un beneficio monetario para los productores de energías limpias. Estos bonos de carbono los compran empresas que emiten gases contaminantes para reducir su nivel registrado de contaminación y poder cumplir con sus compromisos ambientales).

\section{CONCLUSIONES}

Como se ha indicado anteriormente, con objeto de limitar el efecto invernadero en la producción de energía es necesario que el estado implemente políticas para promover el desarrollo de energías limpias como la energía solar. Estas políticas pueden incluir subsidios que cubran las externalidades ambientales de las energías tradicionales o también que el estado gestione ante la comunidad internacional que esta combinación hidroeléctrica con energía solar sea elegible para obtener bonos de carbono. 


\section{REFERENCIAS}

[1] García, R., Nario, T. y Pérez-Reyes, R. (enero del 2011). Valorización de las externalidades y recomposición del parque óptimo de Generación Eléctrica. Organismo Supervisor de la Inversión en Energía y Minería. (28), 26. Recuperado de: http://www.osinergmin. gob.pe/seccion/centro_documental/Institucional/ Estudios_Economicos/Documentos_de Trabajo/Documento_de_Trabajo_28.pdf

[2] Dammert, Alfredo y Raúl García. Economía de la energía. Fondo Editorial PUCP, Lima, Perú, 2017.

[3] Dammert, Alfredo, Juan José Javier Jara y Liz Bautista. El futuro de las energías renovables en el Perú, Fondo Editorial USMP, Lima, Perú, en preparación.

[4] International Energy Agency (IEA). Energy Technology Perspectives 2012, 2012, ParisFrance.

[5] Lopez Ibor, Vicente (ed.) Clean Energy Law and Regulation, Wildy Simmons and Hill Publishing, London, 2017.
[6] Osinergmin. La Industria de la Energía Renovable en el Perú. 2017, Lima-Perú.

[7] www.eea.europa.eu www.sector electricidad. com/109/proyecto-de-la-central-hidroeléctricade-inambari-2000mw/ www.hrudnick.sistios. ing.uc.cl/alumno12/costosernc/C._Hidro.html

[8] Evolución de Costos ERNC www.maps.nrel. gov/rede-peru/? Web del Proyecto PNUMA, MINEM www.pv-magazine-latam.com

[9] Helbling, Thomas (2010) ¿Qué son las externalidades? En: Finanzas y Desarrollo. Fondo Monetario Internacional. Washington DC

[10]Diario Gestión (14 de marzo del 2013). Disney adquirió US\$ 3.5 millones en bonos de carbono en San Martín. Lima, Perú. Recuperado de: https://gestion.pe/economia/ empresas/disney-adquirio-us-3-5-millonesbonos-carbono-san-martin-33948-noticia/ 